\title{
BEWERTUNG DES TEXTVERSTEHENS MIT DEM EUROPÄISCHEN SPRACHENPORTFOLIO
}

\section{Vorbemerkungen}

Im vorliegenden Beitrag wird versucht, auf die Problematik der Selbstbewertung beim Fremdsprachenlernen einzugehen. Die objektive Selbstbewertung der Lernergebnisse ist eines der Ziele, die in allen Lehr- und Lernprozessen gefördert werden sollten. Dieses Ziel wird auch in der Sprachpolitik der Europäischen Union formuliert, u.a. im Gemeinsamen europäischen Referenzrahmen für Sprachen: lernen, lehren, beurteilen und im Europäischen Sprachenportfolio.

Im Beitrag hat man vor, ausgewählte Einsatzmöglichkeiten dieser Dokumente beim Fremdsprachenlernen und ihre Funktionen bei der Entwicklung der Selbstbewertung von rezeptiven Sprachfertigkeiten zu schildern.

\section{Referenzrahmen für Sprachen}

Zur Entstehung des Gemeinsamen europäischen Referenzrahmens für Sprachen: lernen, lehren, beurteilen hat die dreißigjährige intensive Zusammenarbeit vieler Experten in Europa geführt. Die Europäische Union und der Europarat haben die Anstrengungen verschiedener Länder, Institutionen und Spezialisten unterstützt, und als Ergebnis ist das Dokument in einigen Sprachen veröffentlicht worden (u.a. 2000 Englisch, Französisch, 2001 Deutsch, 2003 Polnisch).

Der Gemeinsame europäische Referenzrahmen für Sprachen [...], der ein aktuelles methodisches Kompendium ist, ist das Ergebnis der Bestrebungen der Europäischen Union im Rahmen der Sprachpolitik. Der Europarat verfolgt das Ziel, größere Einheit unter seinen Mitgliedstaaten zu erreichen, was in den Prinzipien des Ministerkomitees des Europarates formuliert wird und bestimmte Initiativen hervorruft (Trim u.a. 2001: 14-15). Die Vielfalt der Sprachen und Kulturen in Europa betrach- 
tet man als einen gemeinsamen Schatz, der in eine Quelle gegenseitiger Bereicherung und gegenseitigen Verstehens umgewandelt werden soll. Dem Verstehen dient auch die Mobilität in Europa, die ziemlich stark mit der Notwendigkeit verbunden ist, moderne europäische Sprachen zu kennen und mehrsprachig zu sein. Daraus resultiert eine Empfehlung, in Europa ein effektives System für den Informationsaustausch über alle Aspekte des Sprachenlernens und -lehrens zu schaffen, was eben der Gemeinsame europäische Referenzrahmen für Sprachen [...] verwirklicht. Besonders wichtig scheint das Ziel zu sein, mit diesem Dokument die Mehrsprachigkeit in Europa zu fördern. Die Autoren verstehen die Mehrsprachigkeit als eine allgemeine kommunikative Kompetenz des Menschen, die sich während des ganzen Lebens durch jede sprachliche und interkulturelle Erfahrung entwickelt. In verschiedenen Situationen können Menschen flexibel auf beliebige Teile dieser Kompetenz zurückgreifen, um eine effektive Kommunikation mit einem bestimmten Gesprächspartner zu erreichen (Trim u.a. 2001: 17).

Das in ganz Europa anerkannte Dokument spielt eine äußerst wichtige Rolle beim Fremdsprachenlehren und -lernen. Es bildet eine Grundlage in zahlreichen Bereichen, die direkt oder indirekt mit dem Erwerb und Lernen von Fremdsprachen zusammenhängen.

Ein Kernelement des Gemeinsamen europäischen Referenzrahmens für Sprachen [...] stellt die Beschreibung der sog. Referenzniveaus der kommunikativen Kompetenz dar (Helbig-Reuter 2004a: 104). Die im Dokument definierten Kompetenzniveaus ermöglichen, sprachliche Leistungen in ganz Europa zu vergleichen sowie Lernfortschritte lebenslang und auf jeder Stufe des Lernprozesses zu messen. Das 6-stufige System soll helfen, die Barrieren zu überwinden, die aus den Unterschieden zwischen den Bildungssystemen in Europa entstehen (Trim u.a. 2001: 14). Es ermöglicht auch die Vergleichbarkeit verschiedener Curricula, Lehrwerke, Prüfungen, Zertifikate und Leistungen beim Fremdsprachenlernen. Heutzutage bedienen sich viele Adressatengruppen dieses Systems. Prüfungsanbieter, Lehrwerkautoren, Institutionen, Behörden, Lehrende und Lernende berufen sich auf die Beschreibungen der einzelnen Stufen im System. Die Bezeichnungen A1, A2, B1, B2, C1 und $C 2^{1}$ werden von immer mehr Menschen mit dem Fremdsprachenlehren und -lernen assoziiert. Diese Entwicklungstendenz lässt hoffen, dass sich das System auch unter Nicht - Fachleuten verbreitet (z.B. unter Arbeitgebern), was sehr hilfreich sein kann, wenn man die Transparenz verschiedener Qualifikationen analysieren will. Mit dem Dokument hat man gemeinsame europäische Standards gewonnen und eine einheitliche Terminologie erarbeitet, die die Kommunikation erleichtert (Quetz 2001: 553).

${ }^{1}$ Die Beschreibung der einzelnen Stufen der Sprachbeherrschung, die sog. Globalskala, ist u.a. im Gemeinsamen europäischen Referenzrahmen für Sprachen: lernen, lehren, beurteilen 2001: 35 zu finden. Die Beschreibung der Referenzniveaus bieten auch zahlreiche WWW-Seiten (u.a. www.codn.edu.pl - polnischsprachige Fassung der Globalskala) an. 
Der Gemeinsame europäische Referenzrahmen für Sprachen [...] bietet eine Grundlage für die erweiterte und differenzierte Leistungsbeurteilung. Er ermöglicht eine Einschätzung der gesamten komplexen fremdsprachlichen und interkulturellen Kommunikationsfähigkeit und berücksichtigt nicht nur die Fremdbewertung, sondern auch die Selbstbewertung. Die Fähigkeit zur objektiven Selbstbewertung ist im Rahmen des lebenslangen Fremdsprachenlernens sehr wichtig, weil sich die Kompetenzentwicklung nicht nur in Schulen und Institutionen realisiert, sondern auch von Lernern individuell beabsichtigt wird, was natürlich auch einer Bewertung, in diesem Fall der Selbstbewertung, bedarf. Sehr behilflich sind deswegen das Raster zur Selbstbeurteilung (Trim u.a. 2001: 36) und zahlreiche Kriterien für die Bewertung der Teilkompetenzen (z.B. Beurteilungsraster zur mündlichen Kommunikation 2001: 37-38, kreatives Schreiben 2001: 67, Hörverstehen allgemein 2001: 71-72, zur Orientierung lesen 2001: 75, Konversation 2001: 80-81, informelle Diskussion 2001: 81-82, Informationsaustausch 2001: 84).

Der Förderung der Mehrsprachigkeit und des verantwortungsvollen Lernens dient auch das zweite Instrument des Europarates, das Europäische Sprachenportfolio, wo unterschiedliche Arten des Sprachenlernens und der interkulturellen Erfahrungen dokumentiert und formell anerkannt werden können.

\section{Sprachenportfolio}

Das Europäische Sprachenportfolio orientiert sich völlig an dem Gemeinsamen europäischen Referenzrahmen für Sprachen [...]. Das Sprachenportfolio ist eine strukturierte Sammlung von Dokumenten unterschiedlicher Art und von Beispielen persönlicher Arbeiten, die von den Lernenden zusammengestellt wird und die sie immer wieder ergänzen und aktualisieren, um ihre Mehrsprachigkeit, ihre Kompetenzen in verschiedenen Sprachen, ihr Sprachenlernen, ihre Sprachkontakte und ihre interkulturellen Erfahrungen für sich selbst und für andere transparent zu dokumentieren (Helbig-Reuter 2004a: 104). Das Europäische Sprachenportfolio ist ein Instrument, mit dem Lernende ihre sprachlichen Fähigkeiten und kulturellen Erfahrungen international vergleichen können. Für das Sprachenlernen als lebensbegleitenden Prozess ist es von zentraler Bedeutung, dass Lernende selbst Verantwortung für Zielsetzung, Fortschritt und Reflexion ihres persönlichen Lernprozesses übernehmen. Optimale Möglichkeiten bietet dazu das Europäische Sprachenportfolio als lernbegleitendes Instrument für jede Altersstufe.

Das Europäische Sprachenportfolio gehört dem Lerner und begleitet ihn beim Sprachenlernen in und außer der Schule. Als Lernbegleiter hat das Portfolio viele Vorteile, denn es bildet eine Basis für die Koordination der Arbeit in verschiedenen Schulfächern, was zur Entwicklung der Lernerautonomie beitragen kann, und es unterstützt die Lerner dabei, über Erfolge und Misserfolge ihres Lernens nachzudenken, bewusst weitere Lernwege zu planen und ihre Leistungen selbst einzu- 
schätzen (Helbig-Reuter 2004b: 174). Jedes dieser Ziele ist sehr wichtig im Lernprozess und kann den Lernern großen Nutzen bringen.

Gemäß den internationalen Richtlinien des Europarates gliedert sich jedes Europäische Sprachenportfolio in drei Teile. Der Sprachenpass gibt Auskunft über den Besitzer und stellt seine Selbsteinschätzung in bestimmten Zeitabständen dar. Hier werden für alle Sprachen jene Fähigkeiten und Kenntnisse dokumentiert, über die der Inhaber bzw. die Inhaberin des Passes verfügt. Diese Fähigkeiten und Kenntnisse werden differenziert nach den international anerkannten Kompetenzstufen (Referenzniveaus) des Europarats $(\mathrm{A} 1-\mathrm{C} 2)$ beschrieben. Darüber hinaus enthält er Angaben über erworbene Zertifikate und Zeugnisse, über Art und Dauer des Sprachenlernens, zu Auslandsaufenthalten und zu Erfahrungen im interkulturellen Lernen.

In der Sprachenbiografie können Lernende die Entwicklung ihrer sprachlichen und kulturellen Kenntnisse und Fähigkeiten aufzeichnen. Hier wird der Verlauf ihrer Lernprozesse beschrieben und reflektiert. Die Sprachenbiografie dient besonders dazu, die eigenen Sprachkenntnisse selbst einzuschätzen und sich gleichzeitig neue Lernziele zu setzen (sog. Checklisten). Hier werden Lerntipps geboten, die bei der Erreichung dieser Ziele helfen können.

Das Dossier ist der dritte Teil des Europäischen Sprachenportfolios. Es ist ein Ordner, wo man durchschnittliche oder auch besonders gelungene Arbeiten sammelt, die den aktuellen Sprachstand oder den Fortschritt dokumentieren sollen. Der Besitzer bestimmt selbst die Art der Arbeit und begründet seine Wahl. Er entscheidet auch, wem und wann er die Sammlung zeigen will.

Länderübergreifende Aufgaben, die in koordinierter Form gestaltet werden sollten, sind die Einführung des Portfolios, die Evaluation der Arbeit mit dem Dokument und seine Weiterentwicklung. Über den Erfolg des Europäischen Sprachenportfolios entscheiden die Benutzergruppen, da sie einen großen Einfluss auf den Prozess seiner Implementierung haben. Von ihnen hängt es ab, ob dieses Dokument eine Chance bekommt, den Lernern auf dem Weg zur Autonomie zu helfen und wie es sich verbreitet. Die Lehrer, die sich als Lernberater verstehen und die die Lerner befähigen wollen, eigene Ziele, Wege und Methoden für sich festzulegen, sollten den Lernenden systematisch das Europäische Sprachenportfolio präsentieren. Die Schüler müssen den Eindruck gewinnen, dass die Arbeit mit diesem Dokument einen integrativen Bestandteil ihres Fremdsprachenlernens darstellt und sich aus dieser Arbeit für ihren persönlichen Lernweg nachvollziehbare Konsequenzen ergeben (Helbig-Reuter 2004b: 174-175).

\section{Bewertung des Textverstehens}

Das Ziel des Fremdsprachenunterrichts ist die Vermittlung der Fremdsprache, um die Lernenden zur Kommunikationsfähigkeit in dieser Fremdsprache zu führen, d.h. zu der Fähigkeit, fremdsprachliche Texte zu verstehen und zu produzieren 
(Solmecke 1993: 7). Diese Aufgabe ist sehr komplex, und deswegen muss sie sorgfältig geplant werden. Jeder Lehrer kann verschiedene Maßnahmen ergreifen und Wege finden, die seine Lerner an dieses Ziel heranführen können.

Im Kommunikationsprozess braucht man unterschiedliche Fertigkeiten. Einerseits sind das rezeptive Fähigkeiten, die es einem erlauben, begegnete Hör- und Lesetexte global, selektiv oder detailliert zu verstehen. Andererseits handelt es sich um produktive Fertigkeiten, die man braucht, wenn man sich schriftlich oder mündlich äußern will. Alle Fertigkeiten können isoliert oder kombiniert entwickelt werden. In der realen Kommunikation im Alltag begegnet man häufig kombinierten Fertigkeiten, z.B. man liest verschiedene Texte zum Thema Umweltschutz und macht sich Notizen, um später an einer Diskussion darüber teilnehmen zu können. In der didaktischen Planung sollte man aber auch die Entwicklung der einzelnen, isolierten Fertigkeiten berücksichtigen, darunter die Entwicklung der Rezeption von Texten.

Texte sind Gegenstand des Hörens und Lesens. Unter „Text“ versteht man eine zusammenhängende mündliche oder schriftliche, monologische oder dialogische Äußerung, die gewöhnlich mehr als einen Satz umfasst und die ein Ziel realisiert, z.B. informiert, beeinflusst, zu etwas veranlasst, Emotionen hervorruft, unterhalten kann (Solmecke 1993: 9). Die Qualität der Texte bestimmt den Grad des Textverstehens, aber sehr wichtig sind in diesem Prozess die mit den Rezipienten verbundenen Faktoren, z.B. ihre Sprachkenntnisse, ihr Vorwissen, ihre Antizipationsfähigkeit, ihre Behaltensmöglichkeiten, ihre Hör- und Lesestrategien. Die Aufgabe des Lehrers ist es, geeignete Texte zu finden, passende Übungen zu wählen und Verstehensstrategien zu fördern. Bei der Wahl der Texte sollten die Lehrkräfte den Umfang, die Textsorte, die thematische Eignung und Schwierigkeitsgrad des/der Texte/s berücksichtigen. Die Übungsgestaltung wird durch die Art und die Reihenfolge der Übungen determiniert, die der Lehrer vorbereitet und die der Evaluation des Verstehens dienen. Der Lehrer sollte den Lernenden auch wirkungsvolle Strategien zeigen, die ihnen helfen sollen, Texte zu verstehen.

Das Europäische Sprachenportfolio bietet eine bedeutende Hilfe bei der Bewertung der Sprachleistungen. Den Ausgangspunkt bilden die Globalskala und das Raster zur Selbstbeurteilung. Eine differenzierte Selbstbewertung jeder Fertigkeit auf allen Stufen der Sprachkompetenz ermöglichen die sog. Checklisten. Aus den Checklisten ergeben sich für die Lernenden neue Rollen: Sie übernehmen nicht nur eine stärkere Verantwortung für ihren Lernprozess, sondern spielen, durch Selbsteinschätzung und durch fortlaufende Reflexion ihres Lernfortschritts, eine aktivere Rolle im Evaluierungsprozess (Glaboniat u.a. 2006: 16).

Jugendlichen Lernern in Polen steht eine speziell für diese Altersgruppe entworfene Portfolioversion ${ }^{2}$ zur Verfügung (Bartczak u.a. 2006). Neben den Checklisten für das Hör- und Leseverstehen findet hier der Lerner Techniken, wie er diese

${ }^{2}$ Im vorliegenden Text wird die Abkürzung ESP 16+ verwendet. 
Fertigkeiten ausbauen und Hinweise, wie er seine Autonomie beim Fremdsprachenlernen entwickeln kann.

In jeder Checkliste befinden sich 8-10 Deskriptoren, die dem Lerner die Selbstbewertung der einzelnen Sprachfertigkeiten auf allen Stufen ermöglichen. Alle Listen sind natürlich nicht vollständig. Sie können beliebig erweitert werden, was die Selbstbewertung noch stärker unterstützen kann. In jeder Portfolioversion nimmt man an, dass das Ankreuzen der Mehrheit der Deskriptoren in einer Liste (im Teil „,ich kann es“) erlaubt festzustellen, dass dieses Niveau erreicht wurde. Die Auswahl von Deskriptoren auf jeder Stufe der sprachlichen Kompetenz stimmt mit den Vorschriften des Europarates, mit den Bedürfnissen des jeweiligen Landes und mit den Erwartungen, die man dem Adressatenkreis gegenüber stellt, überein.

In der Version ESP 16+ formulieren die Autoren die folgenden Deskriptoren im Bereich A1 (Bartczak u.a. 2006: 41-42) folgendermaßen:

\section{1) für das Hörverstehen}

Ich kann verstehen, wenn mich jemand begrüßt oder sich von mir verabschiedet.

Ich kann einfache Höflichkeitsformeln verstehen.

Ich kann einfache Anweisungen verstehen und ausführen.

Ich kann einfache Fragen verstehen, wenn sie sich auf Personen beziehen (Alter, Adresse, Hobby usw.).

Ich kann einfache Fragen verstehen, wenn sie sich auf meine Umgebung beziehen (Haus, Schule, Hochschule usw.).

Ich kann einfache Fragen oder Informationen darüber verstehen, wo sich ein Gegenstand befindet.

Ich kann eine einfache Wegerklärung verstehen.

Ich kann Mengen-, Zeit-, Preisangaben verstehen.

\section{2) für das Leseverstehen}

Ich kann sehr kurze, einfache Texte mit bekannten Wörtern, Wendungen, Namen verstehen.

Ich kann kurze, einfache Mitteilungen auf Postkarten verstehen.

Ich kann kurze schriftliche Anweisungen verstehen (im Lehrbuch, Computerprogramm usw.).

Ich kann kurze Mitteilungen verstehen, die ich bekomme (SMS, E-Mail).

Ich kann einfache Formulare verstehen, die meine persönlichen Angaben betreffen.

Ich kann einfache Informationsmaterialien verstehen, vor allem wenn sie durch Illustrationen begleitet werden.

Ich kann einfache Überschriften bei Fotos und Illustrationen verstehen. 
Die Kompetenzstufe A2 umfasst das Verstehen kurzer Gespräche über den Alltag, des Themas einer Diskussion, bekannter Wendungen in längeren Aussagen, der Hauptinformationen in Fernsehnachrichten, der Fragen und Informationen an öffentlichen Stellen und in Telefongesprächen. Die Lesefertigkeit charakterisiert auf dieser Stufe das Verstehen von einfachen Texten, Privatbriefen, formellen Briefen, typischen Gebrauchstexten (Informationen in Speisekarten, Fahrplänen, Werbeprospekten), einfachen Pressetexten, Kleininseraten, Gebrauchsanweisungen für Geräte des täglichen Gebrauchs und einfachen Informationen zu vertrauten Themen im Internet (Bartczak u.a. 2006: 46-47).

Das Niveau B1 verlangt von Lernenden weitere Fähigkeiten (Bartczak u.a. 2006: 51-52):

- im Hörverstehen: dem Verlauf eines Gesprächs zu Alltagsthemen folgen können, Informationen über Tatsachen aus dem Alltag verstehen, Hauptgedanken zu vertrauten Themen in Aussagen verstehen, Hauptgedanken in Radio- und Fernsehnachrichten verstehen, einfache technische Informationen und detaillierte Anweisungen verstehen

- im Leseverstehen: Informationen in Gebrauchstexten finden können, Berichte über Ereignisse, Gefühle und Wünsche in privaten Briefen verstehen, Hauptthesen von längeren Presseartikeln verstehen, Tatsachen und Meinungen unterscheiden können, der Handlung einer Erzählung folgen können und den Sinn von Gebrauchsanweisungen verstehen.

Weitere Schwerpunkte bei der Rezeption bilden:

B2 Hörverstehen: Äußerungen über bekannte und neue Tatsachen aus Privatleben, Schule, Universität verstehen, lange Äußerungen zu vertrauten Themen verstehen, Diskussionen verstehen, die von Muttersprachlern geführt werden, die meisten Filme, Fernseh- und Radiosendungen verstehen (Bartczak u.a. 2006: 56);

B2 Leseverstehen: Informationen in verschiedenen Texten finden können, Fachartikel über das eigene Gebiet verstehen, Artikel über aktuelle Probleme verstehen, in denen die Autoren einen bestimmten Standpunkt vertreten, zeitgenössische literarische Prosatexte verstehen (Bartczak u.a. 2006: 59);

C1 Hörverstehen: eine lebhafte Diskussion verstehen, die von Muttersprachlern geführt wird, Vorlesungen, Debatten, längere Redebeiträge, komplizierte technische Informationen sowie die meisten Aufnahmen verstehen (Bartczak u.a. 2006: 61);

C1 Leseverstehen: lange und anspruchsvolle Texte verstehen, den Standpunkt des Autors erkennen, jegliche Korrespondenz verstehen, lange literarische Texte in unterschiedlichen Stilen verstehen, längere technische Anweisungen aus verschiedenen Gebieten verstehen (Bartczak u.a. 2006: 62);

C2 Hörverstehen: Vorträge und Fachpräsentationen verstehen, Ironie erkennen können, alle in Medien präsentierten Äußerungen und Informationen verstehen (Bartczak u.a. 2006: 66);

C2 Leseverstehen: alle Textformen verstehen und kritisch interpretieren können, nicht explizit ausgedrückte Bedeutungen verstehen, im Dialekt geschriebene Texte verstehen (Bartczak u.a. 2006: 67). 
Mit insgesamt 30 Listen von Deskriptoren für alle Fertigkeiten (Hörverstehen, Leseverstehen, Sprechen: an Gesprächen teilnehmen und zusammenhängend sprechen, Schreiben) und für jedes Kompetenzniveau (A1, A2, B1, B2, C1, C2) bietet das ESP $16+$ die Möglichkeit, eigene Leistungen beim Fremdsprachenlernen bewerten zu können.

Obwohl die Hilfe des Lehrers bei der Textwahl und Gestaltung von Übungen zur Textrezeption unentbehrlich ist, sind die Deskriptoren auch ein wichtiger Hinweis für die Lernenden, was bei den jeweiligen sprachlichen Tätigkeiten den Schwerpunkt bildet. Die Lernenden können selbst Ziele zur Realisierung wählen, die sie in den Listen finden. Das Ankreuzen der Mehrheit von Deskriptoren hat für die Lernenden einen motivierenden Charakter. Auf diese Weise können sie feststellen, dass systematische Arbeit an der Fremdsprache erfolgreich sein kann und dass sie einen Fortschritte gemacht haben. Motivationsfördernd ist auch die Tatsache, dass im Portfolio Kenntnisse in allen Fremdsprachen dokumentiert werden, die man lernt. Man betrachtet hier sprachliche Kompetenzen in ihren Wechselwirkungen und stellt die Entwicklung des eigenen sprachlichen Könnens fest.

\section{Abschießende Bemerkungen}

Die rezeptiven Sprachfertigkeiten bilden nur einen Teil der Sprachkompetenz, aber ohne sie kann man sich die Entwicklung der Mitteilungsfähigkeit nicht vorstellen. Das Europäische Sprachenportfolio unterstützt die Entwicklung des ganzen sprachlichen Könnens der Lernenden und macht ihnen das Lernen von Fremdsprachen bewusst. Das alles kann zur Herausbildung eines autonomen Verhaltens der Lerner beitragen, was wiederum einen Nutzen in verschiedenen Lebensbereichen und Lebensphasen haben kann. Deswegen sollte man alle Fremdsprachenlehrer dazu anregen, ihre Schüler mit dem Europäischen Sprachenportfolio vertraut zu machen und ihnen seine Vorteile zu zeigen.

Die Bestrebungen der Lehrkräfte, das autonome Verhalten bei den Lernern zu entwickeln, sollten einen langfristigen Charakter haben, weil die Veränderung der Lerngewohnheiten viel Zeit braucht. Sie können aber den Lernern eine Chance geben, einen großen Erfolg beim Fremdsprachenlernen zu erleben und gleichzeitig vielseitige Fähigkeiten herauszubilden. Von großer Bedeutung ist dabei die Fähigkeit, sich selbst objektiv bewerten zu können, die sehr stark im Europäischen Sprachenportfolio gefördert wird.

\section{Literatur}

Bartczak, E.; Lis, Z.; Marciniak, I.; Pawlak, M. (2006): Europejskie portfolio językowe dla uczniów szkót ponadgimnazjalnych i studentów. Warszawa: Centralny Ośrodek Doskonalenia Nauczycieli 
Glaboniat, M.; Müller, M. (2006): Referenzrahmen und Profile Deutsch in ihren Auswirkungen auf Prüfungen und Tests. In: Fremdsprache Deutsch Nr. 34. München: Langenscheidt, 14-21

Helbig-Reuter, B. (2004a): Das Europäische Portfolio der Sprachen (I). In: Deutsch als Fremdsprache Nr. 2. München: Langenscheidt, 104-110

Helbig-Reuter, B. (2004b): Das Europäische Portfolio der Sprachen (II). In: Deutsch als Fremdsprache Nr. 3. München: Langenscheidt, 173-176

Quetz, J. (2001): Der gemeinsame europäische Referenzrahmen. In: Info DaF Nr. 6. München: IUDICIUM Verlag GmbH, 553-563

Solmecke, G. (1993): Texte hören, lesen und verstehen. Berlin, München, Wien, Zürich, New York: Langenscheidt

Trim, J.; North, B.; Coste, D.; Sheils, J. (2001): Gemeinsamer europäischer Referenzrahmen für Sprachen: lernen, lehren, beurteilen. Berlin, München, Wien, Zürich, New York: Langenscheidt 